\title{
Effectiveness of Strategic Training of Neuro-Linguistic Programming on Improving the Quality of Life of Veterans' Spouses with Injury Rate of 25 to 50 Percent; A Case Study of Tehran Province
}

\section{ART ICLE INF O}

\section{Article Type}

Original Research

\section{Authors}

Fahimi Rad SZ** $M A$,

Salehi GH. ${ }^{1} M A$,

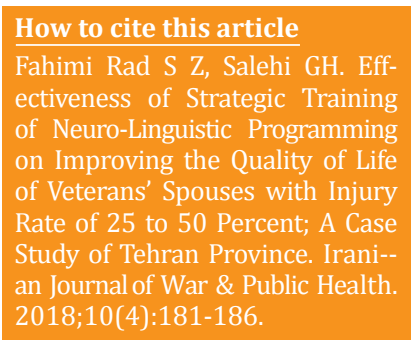

*Counseling Department, Faculty of Psychology and Consultation, University of Social Welfare Sciences, Tehran, Iran

${ }^{1}$ Department of Psychology, Faculty of Psychology and Educational Sciences, Allameh Tabatabaei University, Tehran, Iran

\section{*Correspondence}

Address: Tehran, Narmak, Square 57, Akhtar Alley, No. 9. Postcode 1681773811

Phone: +98 (21) 33770011

Fax: +98 (21) 33372010

zhr_fahimirad@yahoo.com

\section{Article History}

Received: August 30, 2017

Accepted: June 02, 2018

ePublished: October 11, 2018

\section{A B S T R A C T}

Aims Neuro-Linguistic programming refers to specific relationships between neurological processes, linguistic and behavioral patterns learned through planning. The main purpose of the present study was to investigate the effect of training neuro-linguistic programming strategies on improving the quality of life of veterans' wives from $25-50 \%$.

Materials \& Methods In this semi-experimental study, with pre-test post-test design with control group, 30 Veterans wives from 25-50\% in the cities of Tehran province were selected randomly and divided in experimental and control groups (each group of 15 people). The tool used in this study was a short-scale quality of life. The training plan was submitted to the experimental group in 10 sessions. Data analysis was done using statistical software SPSS 23, after the calculation of the difference between the pre-test and post-test scores, the multivariate covariance analysis (Mancova) was used for both groups.

Findings Significant positive changes were observed between the mean scores of the subscales of mental health, physical health, social relationships, perception of the living environment and in general the quality of life of veterans' veterans of the experimental group at the stage of verbal neural planning training $(\mathrm{p}<0.05)$; While this difference was not observed in the control group and in almost all subscales of quality of life in this group (control) were close to each other at the pre and post education stage and they did not show any difference ( $p>0.05)$.

Conclusion Training neuro-linguistic programming strategies improves the quality of life of veterans' wives from $25-50 \%$.

Keywords Quality of Life; Neuro-linguistic programming strategies; Spouses; Veterans

\section{I T A T I O N L I N K S}

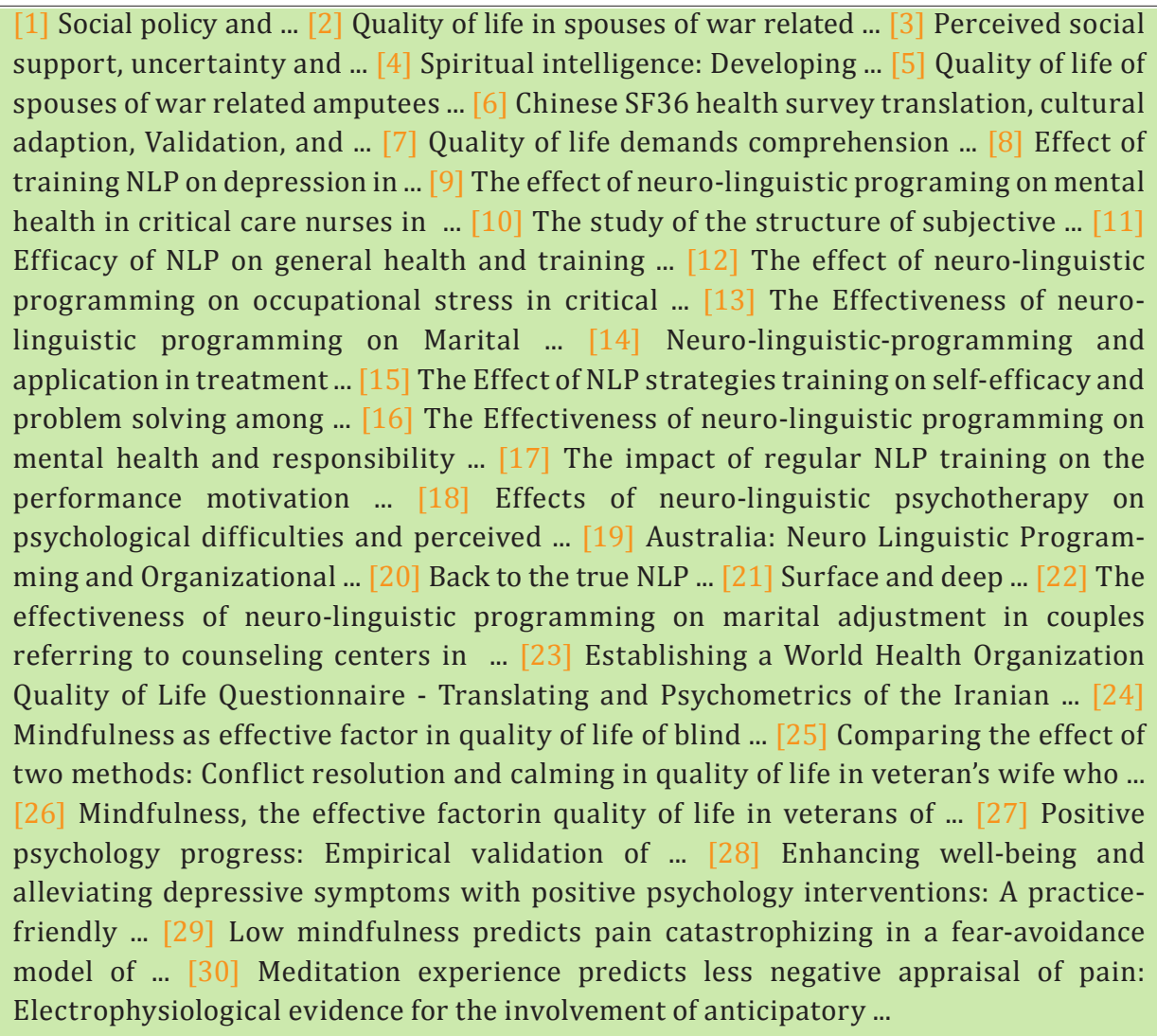


فرد، خانواده و مخصوصاً همسر او به وجود مى آورد[1]. اثرات اين

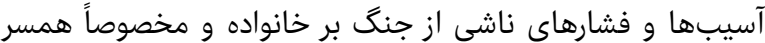

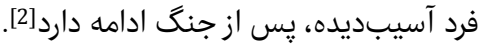

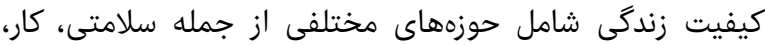

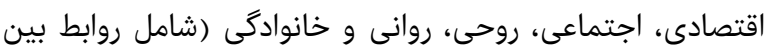

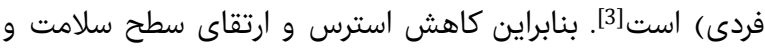

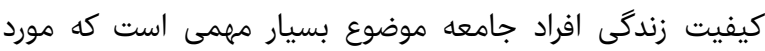
توجه صاحبنظران بهداشتى قرار كرفته است. به اعتئ اعتقاد كترهال،

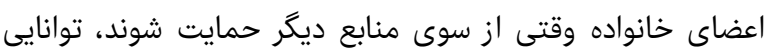

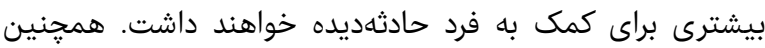

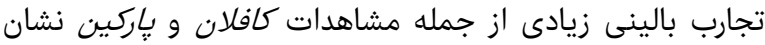

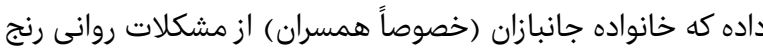

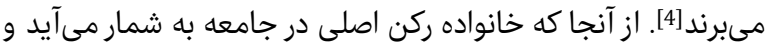

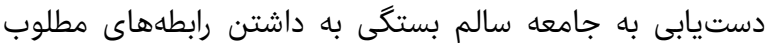

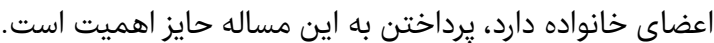

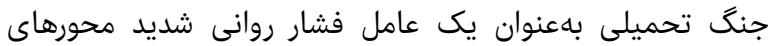

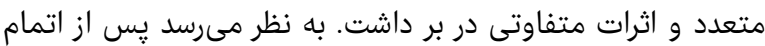

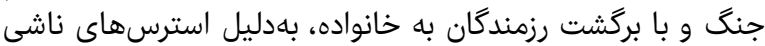

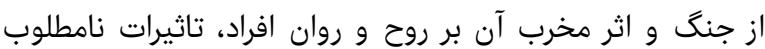

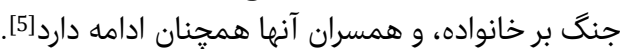

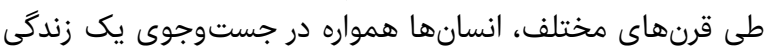

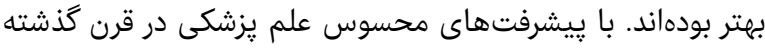

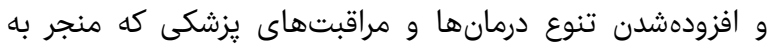

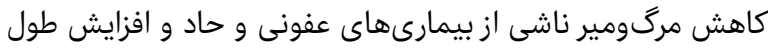

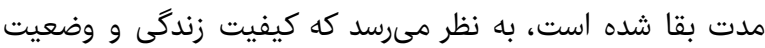

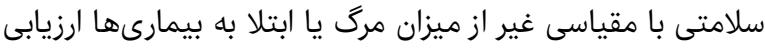

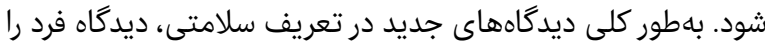

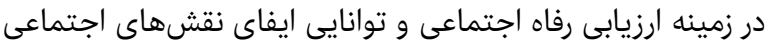

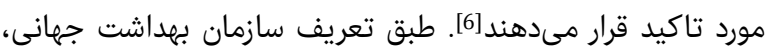

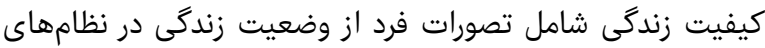

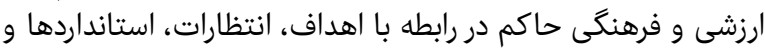

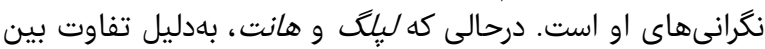

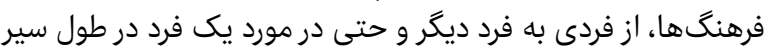

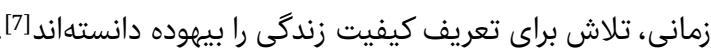

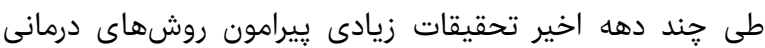

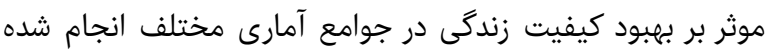

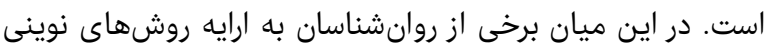

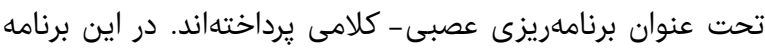

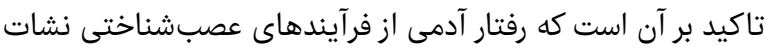

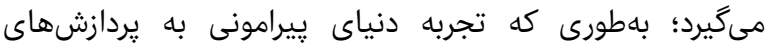

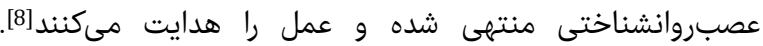

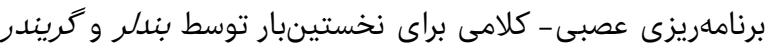

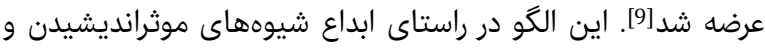

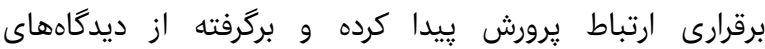

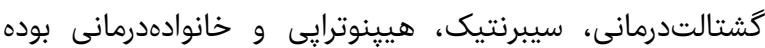

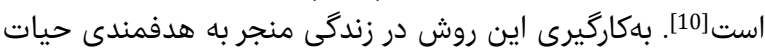

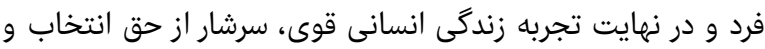

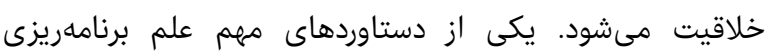

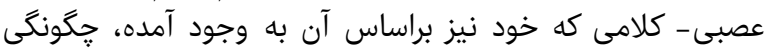

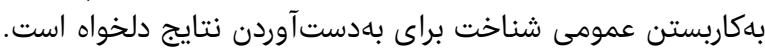

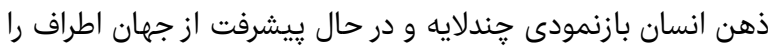

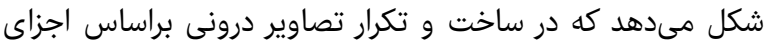

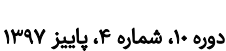

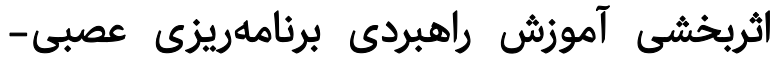

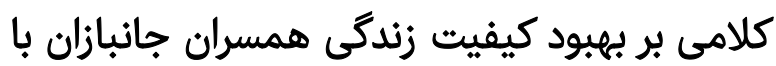

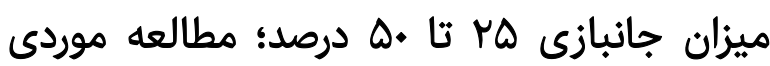
استان تهران

MA " سيدززهرا فهيمىراد كروه مشاوره، دانشكده روانشناسى و مشاوره، دانشكاه علوم بهزيستى، تهران، ايران

غ غلامرضا صالحى ايران كروه روانشناسى، دانشكده روانشناسى و علوم تربيتى، دانشكاه علامه طباطبايى،

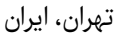

جكيده

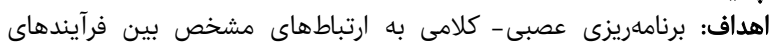

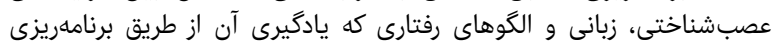

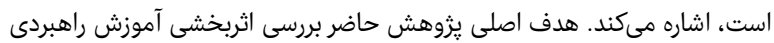

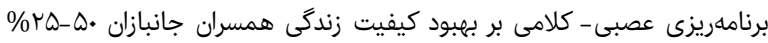

بود.

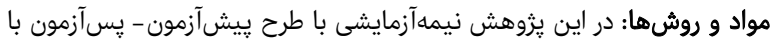

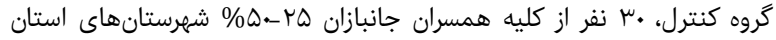

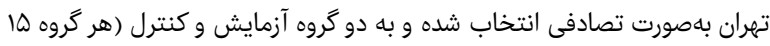

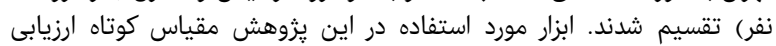

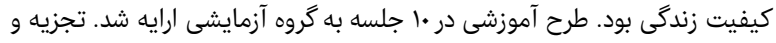

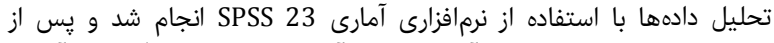

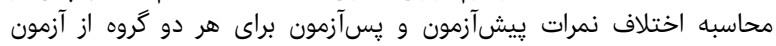

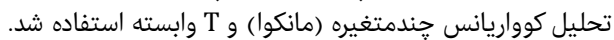

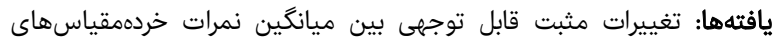

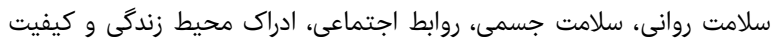

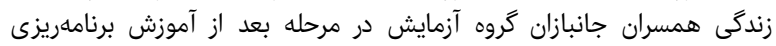

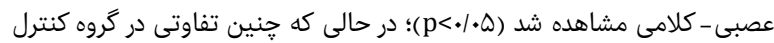

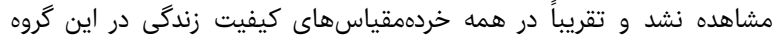

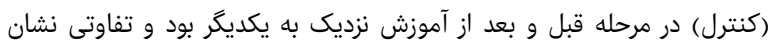

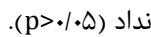
نتيجهكيرى: آموزش ران راهبردى برنامهريزى عصبى- كلامى باعث بهبود كيفيت

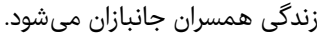

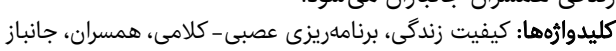

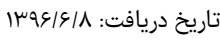

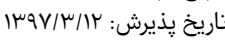

zhr_fahimirad@yahoo.com : تويسنده مسئوش:

مقدمه

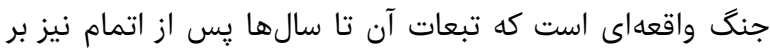

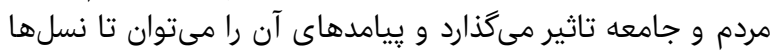

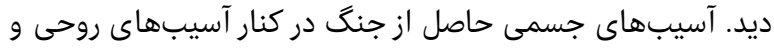

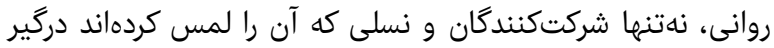

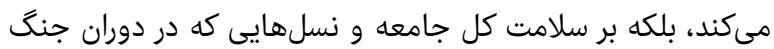

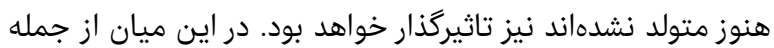

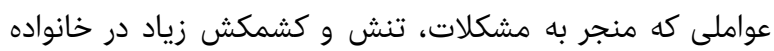

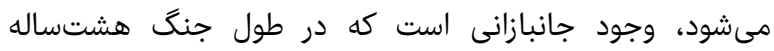

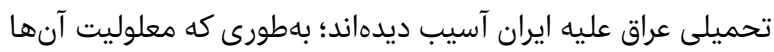

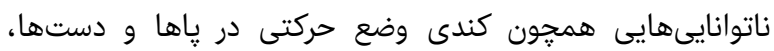

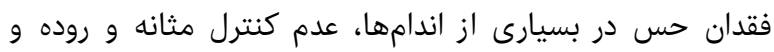

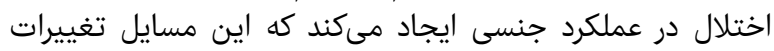

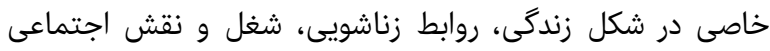




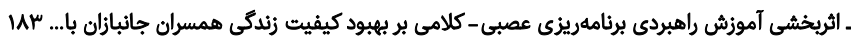

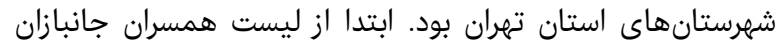

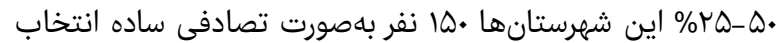

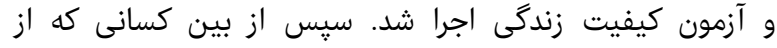

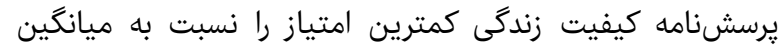

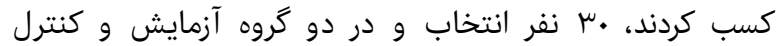

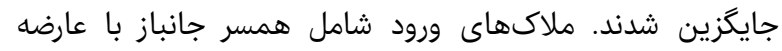

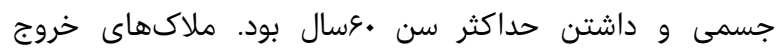

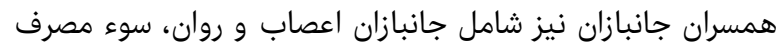
مواد و جانبازان شيميايى بود.

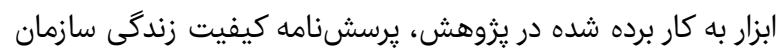

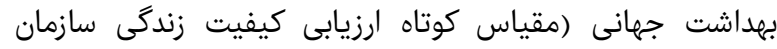

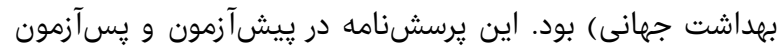

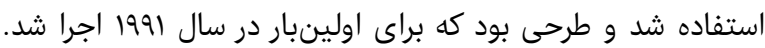

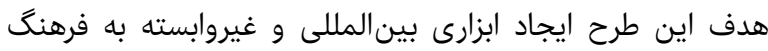

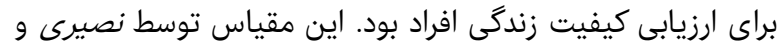

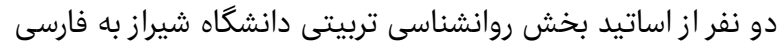

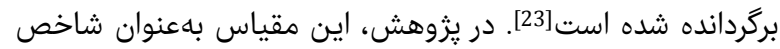

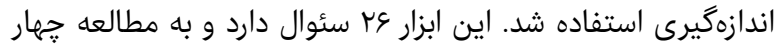

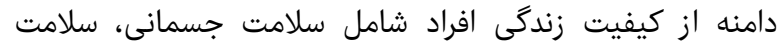

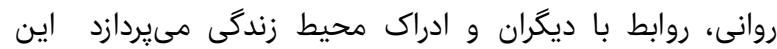

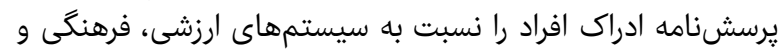

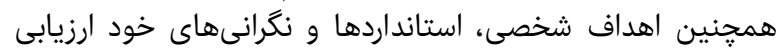

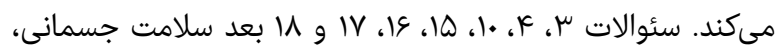

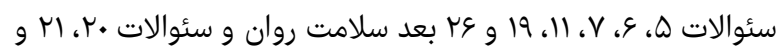

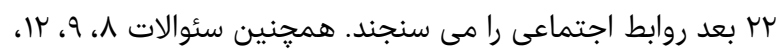

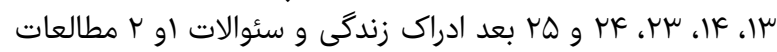

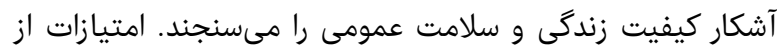

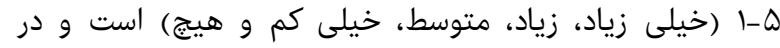

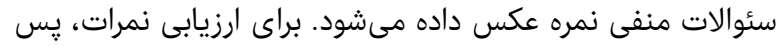

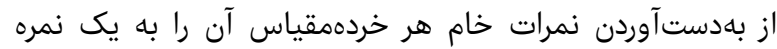

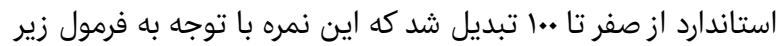

محاسبه شد:

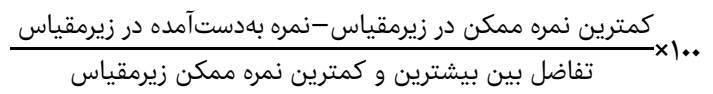

در مورد يايايى مقياس، توسط گروه سازندكان مقياس كيفيت

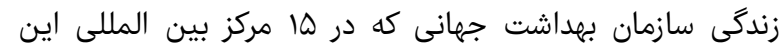

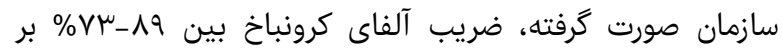

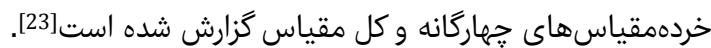

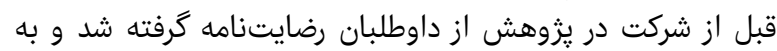

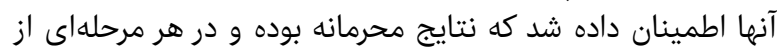

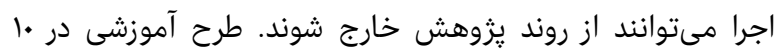

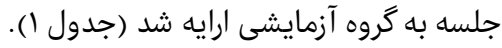

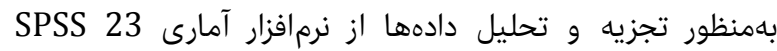

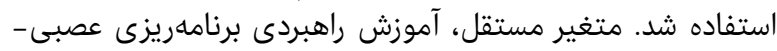

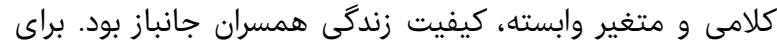

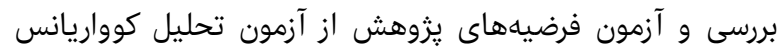

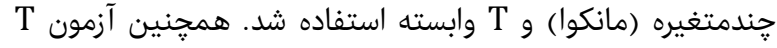

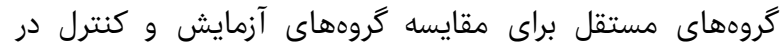

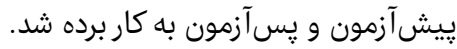

ديدارى، شنيدارى، جنبشى، بويايى، جشايى و شهائ إنهودى بر تجربه

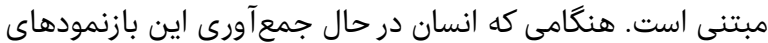

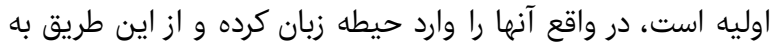

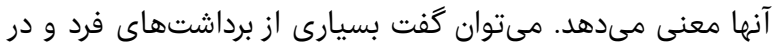

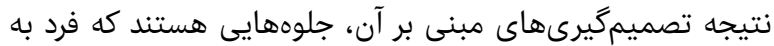

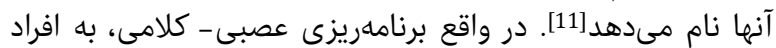

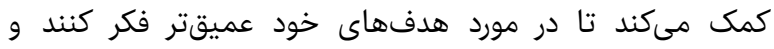
درستترين هدف را در هر موقعيت زمانى انتخاب كرده ور در درو صورت لزوم آن را تغيير دهند [12].

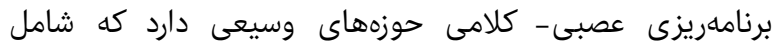

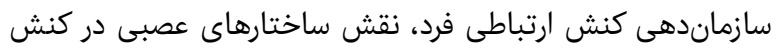

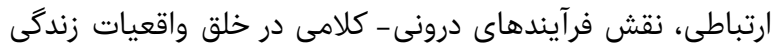

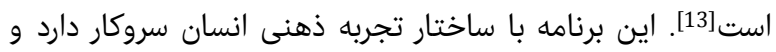

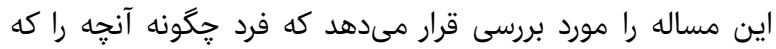

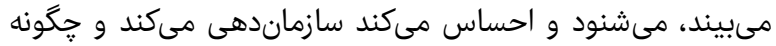

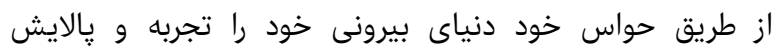

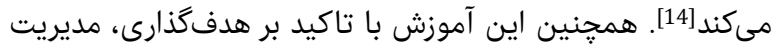

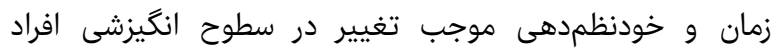

مى شود [15].

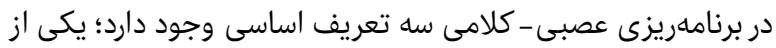

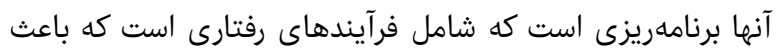

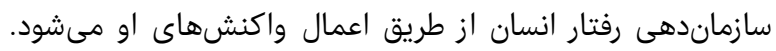

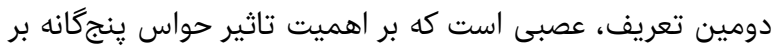

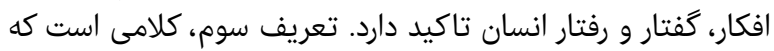

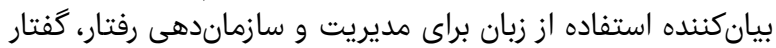

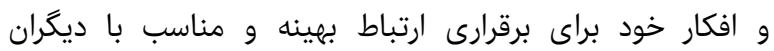

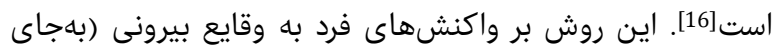

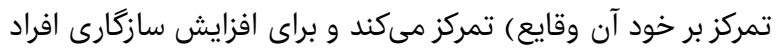

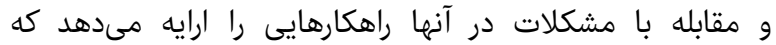

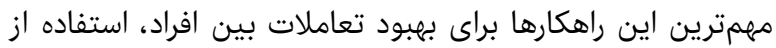

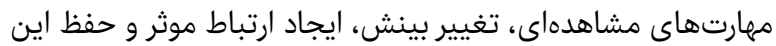

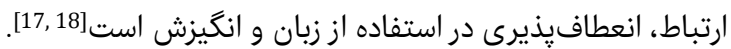

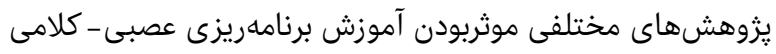

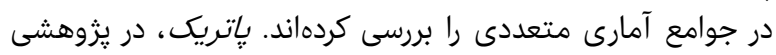

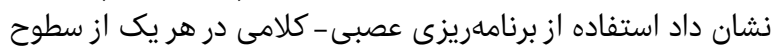

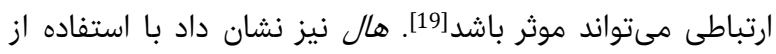

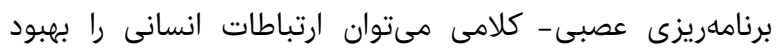

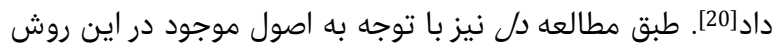

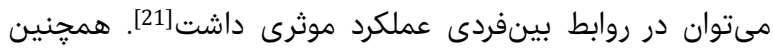

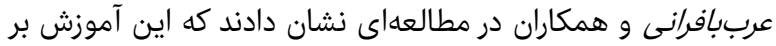

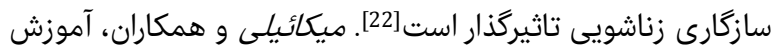

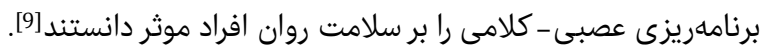

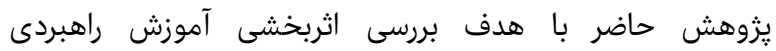

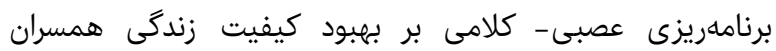

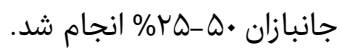

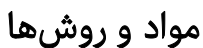

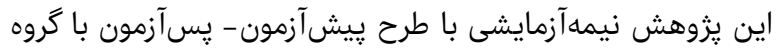

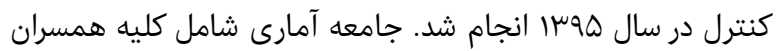

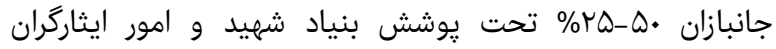




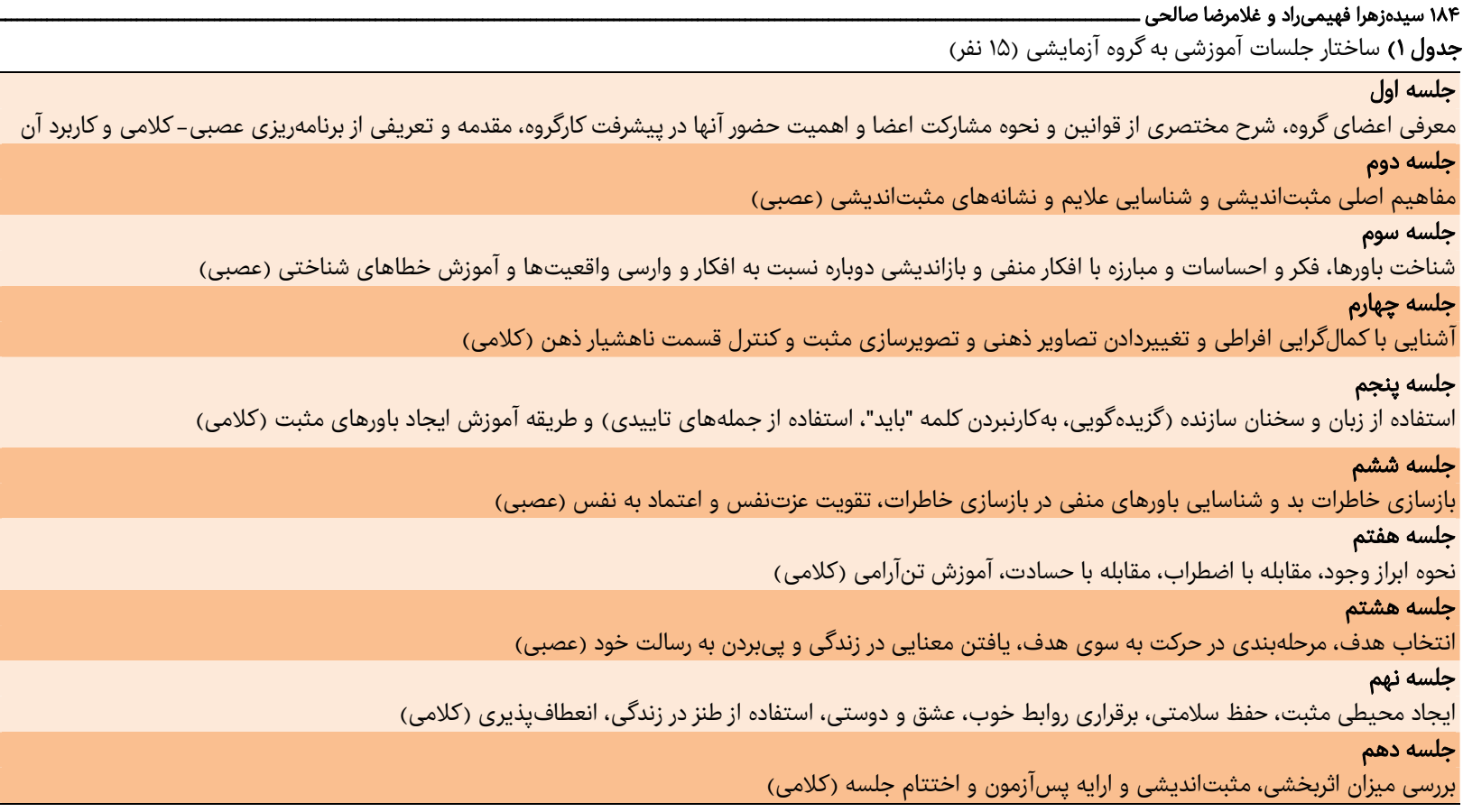

زند

يافتهها زندگى (

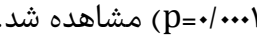

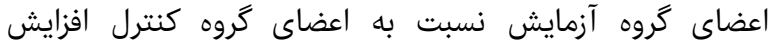

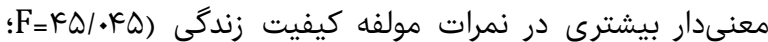
جr

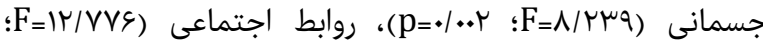

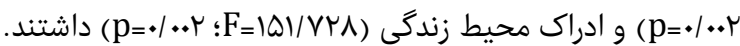

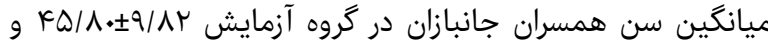

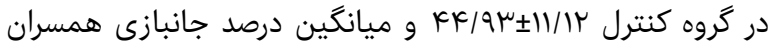

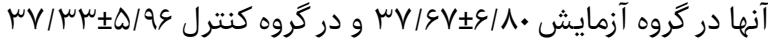

بودر.

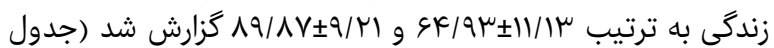

بحث

هدف از اين يزوهش بررسى اثربخشى آموزش راهبردى برنامهريزى

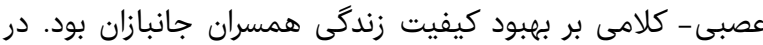

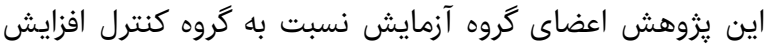

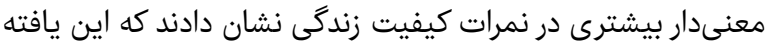

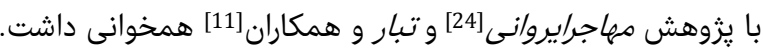

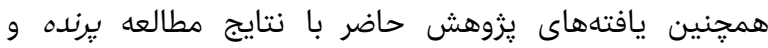

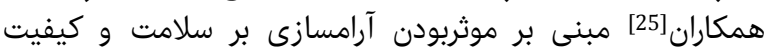

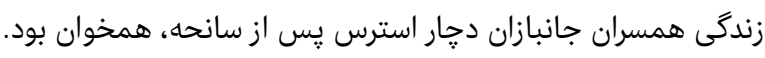

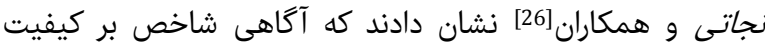

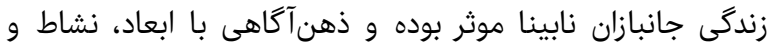

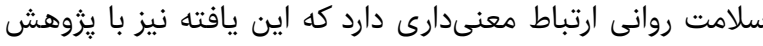
حاضر همخوانى داشت.

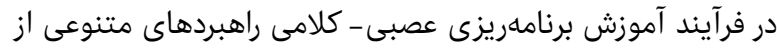

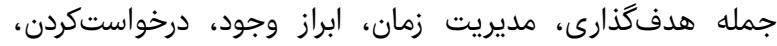

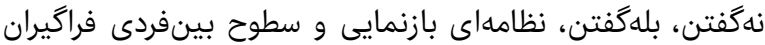

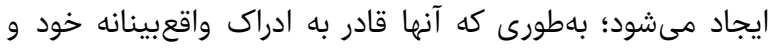

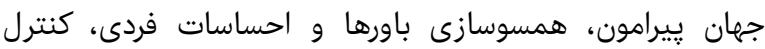

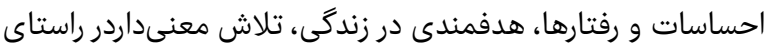

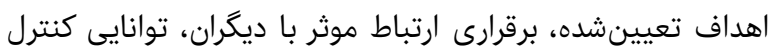

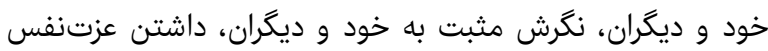

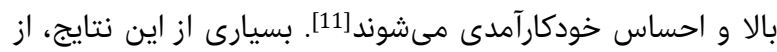

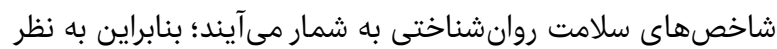

جدول r) ميانگين آمارى نمرات آزمودنىها در گروه آزمايش (Qا نفر) و كنترل

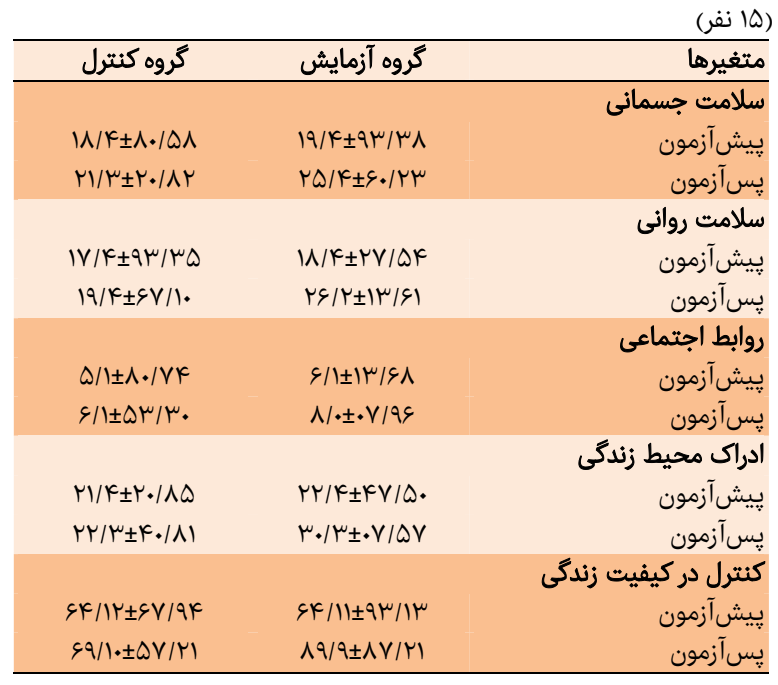

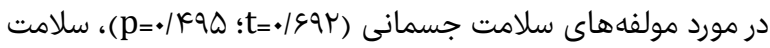

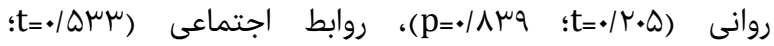

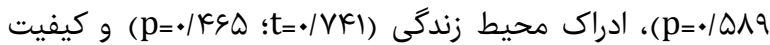

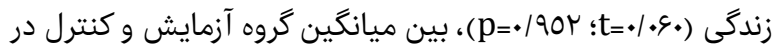

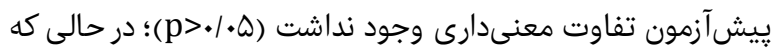

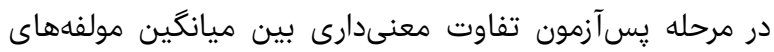

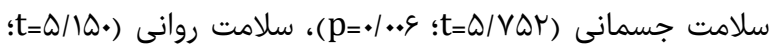




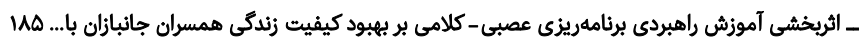

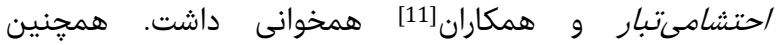

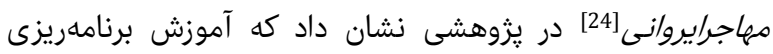

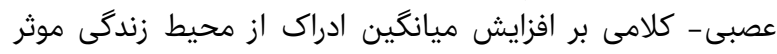

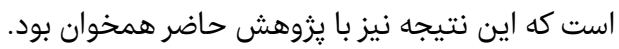

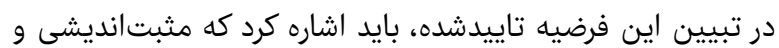

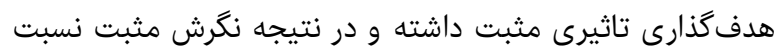

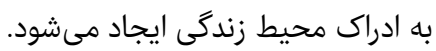

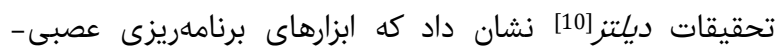

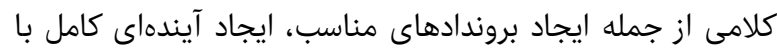

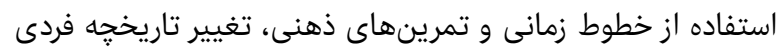

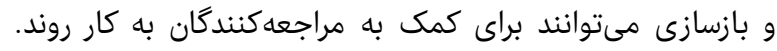

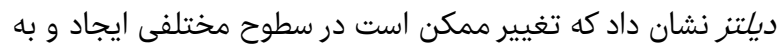

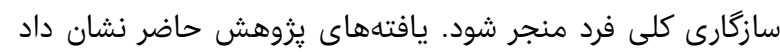

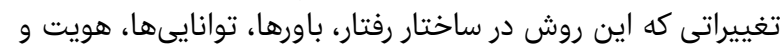

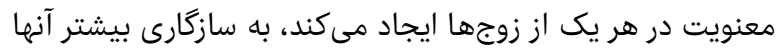

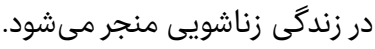

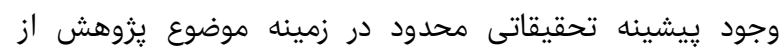

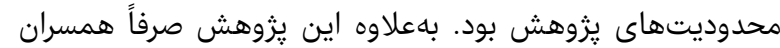

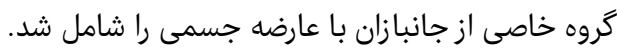

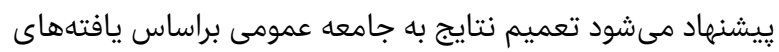

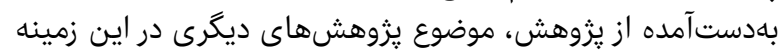

باشد.

\section{نتيجه آمهزيرى}

آموزش راهبردى برنامهريزى عصبى - كلامى باعث بهبود كيفيت

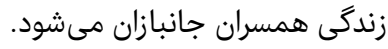

تشكر و قدردانى: از كليه خانوادههاى جانبازان عزيز مخصوصاً

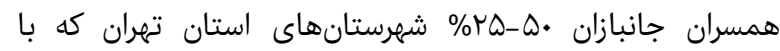
صبورى خود ما را در اين يزوهش همران هراهى كردند كمال سياس و وان

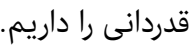

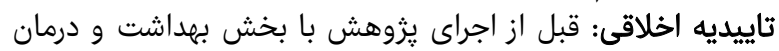

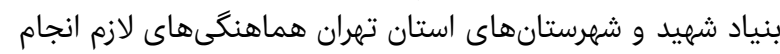

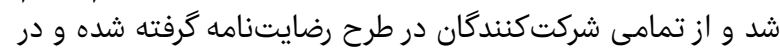

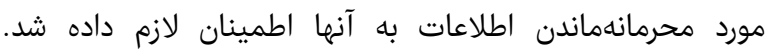

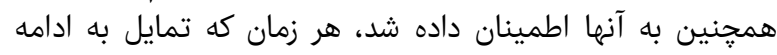

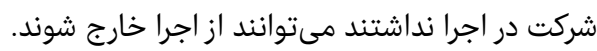

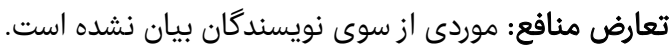

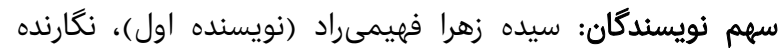

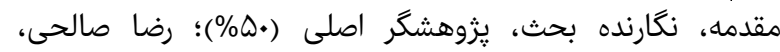

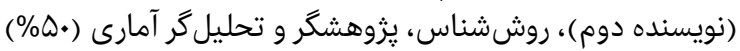

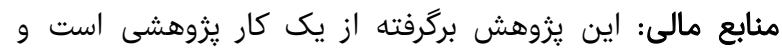

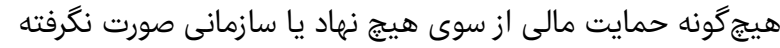

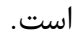

منابع

1- Babai N. Social policy and health. J Soc Welf. 2003;3(10):201-32. [Persian]

2- Mousavi B, Soroush MR, Masoumi M, Ganjparvar Z, Montazeri A. Quality of life in spouses of war related bilateral lower limb amputees. Daneshvar Med. 2010;17(84):11-18. [Persian]

3- Sammarco A. Perceived social support, uncertainty

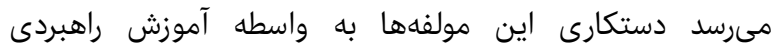
برنامهريزى عصبى- كلامى در بهبود كيفيت زندارى دولى فراگيران موثر واقع شود. در يزوهش حاضر، اعضاى گروه آزمايش نسبت به گروه كنترل

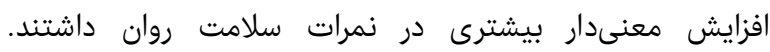

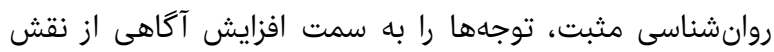

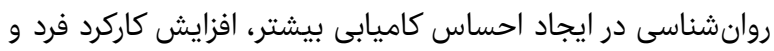

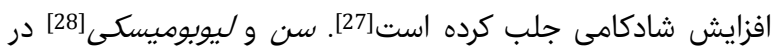

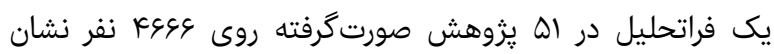

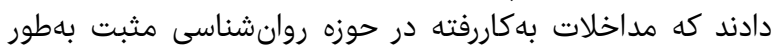

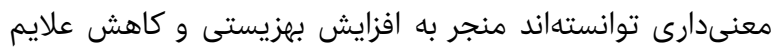

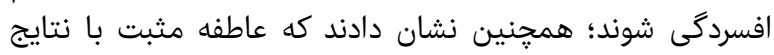

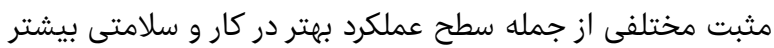

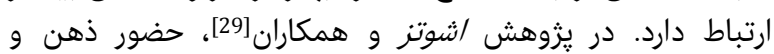

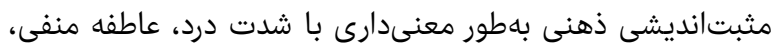

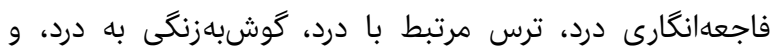

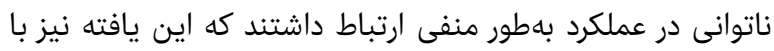

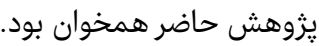

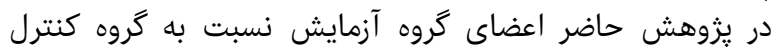

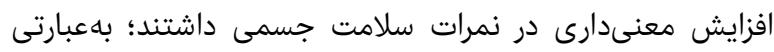

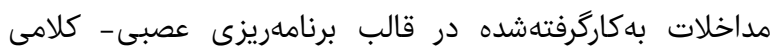

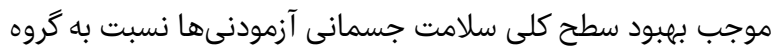

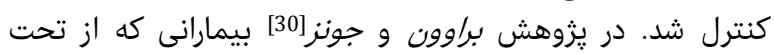

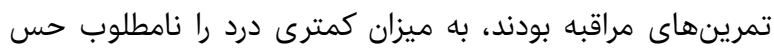

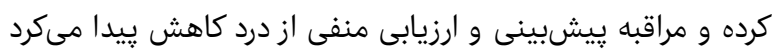

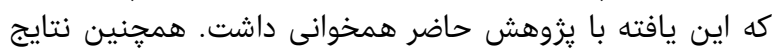

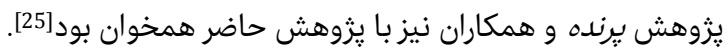

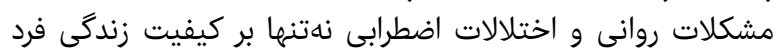

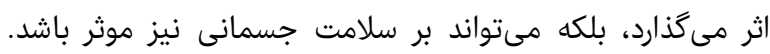

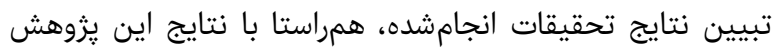

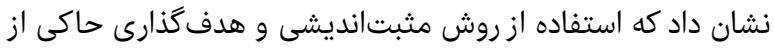

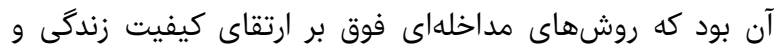
سطح سلامت جسمانى افراد موثر است.

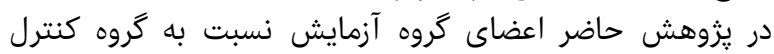

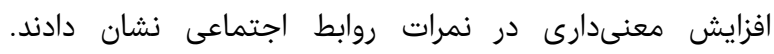

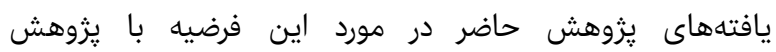

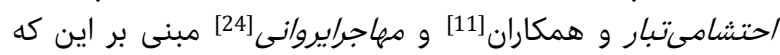

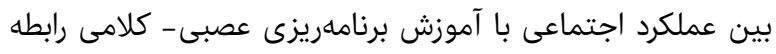

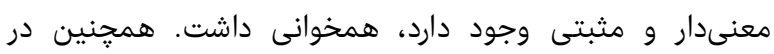

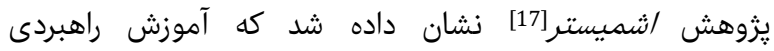

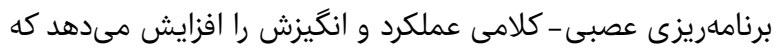

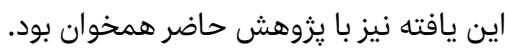

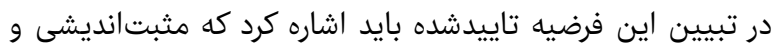

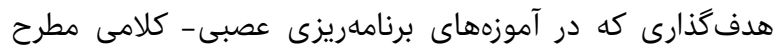

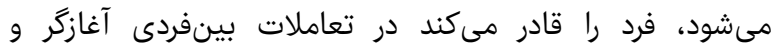

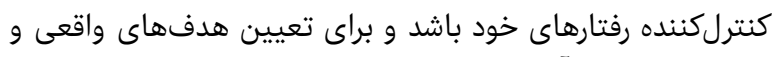

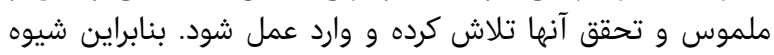

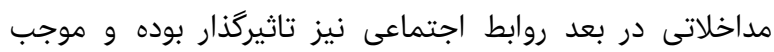

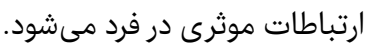

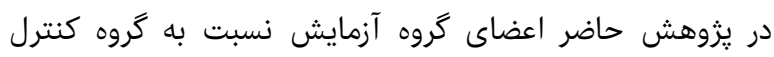

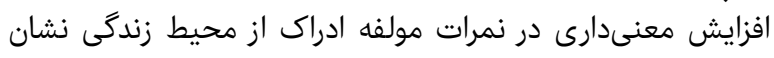

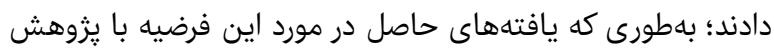


2002. pp. 67-76.

18- Stipancic M, Renner W, Schutz P, Donel R. Effects of neuro-linguistic psychotherapy on psychological difficulties and perceived quality of life. Couns Psychother Res. 2010;10(1):39-49.

19- NLpmax [Internet]. Australia: Neuro Linguistic Programming and Organizational Devlopment University of Sydney; 2007 [cited 2007 Jun 17]. Available from: http: www.nlpmax.com.

20- Hall LM. Back to the true NLP sources. Anchore Point. 2008; $17: 53-5$.

21- Doel S. Surface and deep structure. Anchore Point. 2002;16(12):12-5.

22- Arab bafarani H, Abedi A, Behrouz B, Naghavi M, Ashrafabadi M. The effectiveness of neuro-linguistic programming on marital adjustment in couples referring to counseling centers in Isfahan. J New Psychol Res. 2012;7(28):141-57. [Persian]

23- Nejat S, Montazeri A, Halakoee Naeeni K, Mohammad K, Majdzadeh SR. Establishing a World Health Organization Quality of Life Questionnaire - Translating and Psychometrics of the Iranian Species. J Health Inst Health Res. 2006;4(4):1-12. [Persian]

24- Nejati V, Amini R, Zabihzadeh A, Masoumi M, Maleki Gh, Shoaie F. Mindfulness as effective factor in quality of life of blind veterans. Tebe e Janbaz. 2011;3(11):1-7. [Persian]

25- Parande A, Sirati Nayer M, Khaghanizade M, Karimi Zarchi A. Comparing the effect of two methods: Conflict resolution and calming in quality of life in veteran's wife who suffer from PTSD with depression. J Fundam Ment Health. 2007;9(33-34):67-76. [Persian]

26- Nejati V, Amini R, Zabihzade A, Masoomi M, Maleki G, Shoaee F. Mindfulness, the effective factorin quality of life in veterans of blind. Iran J War Public Health. 2011;3(11):1-7. [Persian]

27- Seligman ME, Steen TA, Park N, Peterson C. Positive psychology progress: Empirical validation of intervention. Am Psychol. 2005;60(5):410-21.

28- Sin LN, Lyubomirsky S. Enhancing well-being and alleviating depressive symptoms with positive psychology interventions: A practice-friendly metaanalysis. J Clin Psychol. 2009;65(5):467-87.

29- Schütze R, Rees C, Preece $M$, Schütze M. Low mindfulness predicts pain catastrophizing in a fearavoidance model of chronic pain. Pain. 2010;148(1):1207.

30- Brown CA, Jones AK. Meditation experience predicts less negative appraisal of pain: Electrophysiological evidence for the involvement of anticipatory neural responses. Pain. 2010;150(3):428-38.

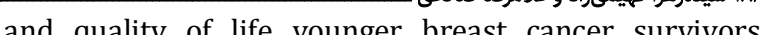
Cancer Nurs. 2001;24(3):212-9.

4- Sisk DA, Torrance EP. Spiritual intelligence: Developing higher consciousness. New York: Consciousness Buffalo; creative Education foundation press; 2001.

5- Naderi A, Sedighi Sh, Roshanae Gh, Ahmadpanah M, Rostampur F, Asadi z. Quality of life of spouses of war related amputees of Hamedan city, Iran. Iran J War Public Health. 2016;8(3):157-63. [Persian]

6- Li L, Wang HM, Shen Y. Chinese SF36 health survey translation, cultural adaption, Validation, and normalization. J Epidemiol Community Health. 2003;57(4):259-63.

7- Bergland A, Narum I. Quality of life demands comprehension and further exploration. J Aging Health. 2007;19(1):39-61.

8- Ahmadi R, Ahadi H, Mazaheri M, Delavar A, Najarian B. Effect of training NLP on depression in student. J New Find Psychol. 2011;6(18):59-74. [Persian]

9- Mikaeeli F, Hemati Maslakpak M, Farhadi M, Feizipour $\mathrm{H}$. The effect of neuro-linguistic programing on mental health in critical care nurses in Urmia. J Urmia Nurse Midwifery Fac. 2016;14(5):437-45. [Persian]

10- Dilts RB. The study of the structure of subjective experience. Santa Cruz: Meta Publications; 1980.

11- Ehteshami Tabar A, Moradi A, Shahraray M. Efficacy of NLP on general health and training motivation. J psychol. 2006;10(1):52-65. [Persian]

12- Hemmati Maslakpak M, Farhadi M, Fereidoni J. The effect of neuro-linguistic programming on occupational stress in critical care nurses. Iran J Nurs Midwifery Res. 2016;21(1):38-44.

13- Kajbaf MB, Moghaddas M, Aghaei A. The Effectiveness of neuro-linguistic programming on Marital Adjustment. J Knowl Res Appl Psychol. 2012;12(4)(continuous No.46):30-40. [Persian]

14- Karunaratne M. Neuro-linguistic-programming and application in treatment of phobias. J Complement Ther Clin Pract. 2010;16(4):203-7.

15- Zamini S, Hosseini Nasab SD, Hashemi T. The Effect of NLP strategies training on self-efficacy and problem solving among girl students. J Psychol. 2009;13(3):25871. [Persian]

16- Saadati Shamir A, Zahmatkesh Z. The Effectiveness of neuro-linguistic programming on mental health and responsibility of bojnourd's students. J Educ Res. 2006;(8):63-77. [Persian]

17- Scheemeister E. The impact of regular NLP training on the performance motivation of children in primary school. 1 ${ }^{\text {st }}$ Edition. Santa Cruz: NLP university press; 